JOURNAL OF

SYMPLECTIC GEOMETRY

Volume 1, Number 2, 427-435, 2002

\title{
Correction: The Verlinde formulas as fixed point formulas
}

\author{
A. Alekseev, E. Meinrenken, and C. Woodward
}

Due to a publisher error, the correct version of sections 5.4, 5.5, and 5.6 was not printed. The correct version is included here,

\section{Verlinde formula.}

\subsection{The fixed point sets.}

The fixed point sets for the action on the holonomy manifold are symplectic tori:

Proposition 5.1. The fixed point set for the action of $\left(t_{\lambda_{1}}, \ldots, t_{\lambda_{r}}\right)$ on $M\left(\Sigma_{h}^{r}\right)=G^{2(h+r-1)}$ is empty unless $\lambda_{1}=\ldots=\lambda_{r}=: \lambda$, and

$$
M\left(\Sigma_{h}^{r}\right)^{\left(t_{\lambda}, \ldots, t_{\lambda}\right)}=F:=T^{2(h+r-1)} .
$$

Proof. Since $M\left(\Sigma_{h}^{r}\right)$ is obtained from a direct product of $h+r-1$ copies of $M\left(\Sigma_{0}^{2}\right)$ by passing to diagonal actions for some of the $G$-factors, it suffices to prove Proposition 5.1 for $\Sigma_{0}^{2}$. By $(28)$, an element $(a, b) \in M\left(\Sigma_{0}^{2}\right)$ is fixed by $\left(t_{\lambda_{1}}, t_{\lambda_{2}}\right)$ if and only if

$$
t_{\lambda_{1}}=\operatorname{Ad}_{a} t_{\lambda_{2}}, \quad t_{\lambda_{2}}=\operatorname{Ad}_{b} t_{\lambda_{1}}
$$

Both $t_{\lambda_{1}}$ and $t_{\lambda_{2}}$ belong to the exponential of the alcove $\exp (\mathfrak{A})$. Since each conjugacy class meet $\exp (\mathfrak{A})$ only once, $(31)$ holds if and only if $\lambda_{1}=\lambda_{2}$.

Notice that the fixed point set is independent of $\lambda$; in fact, $F$ is fixed by the full diagonal torus $T \subset G^{r}$. 


\subsection{Evaluation of the fixed point contributions.}

Let $\Sigma=\Sigma_{h}^{r}$ and $\mu=\left(\mu_{1}, \ldots, \mu_{r}\right)$ with $\mu_{j} \in \Lambda_{k}^{*}$. By Theorem 4.3 , the Spin $_{c}$-index is given by the formula

$$
\chi\left(\mathcal{M}(\Sigma)_{\mu}\right)=\frac{1}{\left(\# T_{k+c}\right)^{r}} \sum_{\lambda \in \Lambda_{k}^{*}} \chi_{\mu_{1}}\left(t_{\lambda}\right) \cdots \chi_{\mu_{r}}\left(t_{\lambda}\right)\left|J\left(t_{\lambda}\right)\right|^{2 r} \chi\left(\nu_{F}, t_{\lambda}\right)^{*}
$$

Here we abbreviated $\left(t_{\lambda}, \ldots, t_{\lambda}\right)$ to $t_{\lambda}$, viewing $T$ as diagonally embedded into $G^{r}$. For the calculation of the fixed point contribution

$$
\chi\left(\nu_{F}, t_{\lambda}\right)=\zeta_{F}\left(t_{\lambda}\right)^{1 / 2} \int_{F} \frac{\hat{A}(F) e^{\frac{1}{2} c_{1}\left(\mathcal{L}_{F}\right)}}{\mathcal{D}_{\mathbb{R}}\left(\nu_{F}, t_{\lambda}\right)},
$$

note first of all that

$$
\hat{A}(F)=1
$$

since $F$ is a torus. Furthermore, since the normal bundle $\nu_{F}$ is $T$ equivariantly isomorphic to $(\mathfrak{g} / \mathfrak{t})^{2(h+r-1)}$, we have

$$
\mathcal{D}_{\mathbb{R}}\left(\nu_{F}, t_{\lambda}\right)=J\left(t_{\lambda}\right)^{2(h+r-1)}=(-1)^{(h+r-1) \# \Re}\left|J\left(t_{\lambda}\right)\right|^{2(h+r-1)} .
$$

It remains to work out the integral $\int_{F} \exp \left(\frac{1}{2} c_{1}\left(\mathcal{L}_{F}\right)\right)$ and to calculate the phase factor $\zeta_{F}\left(t_{\lambda}\right)^{1 / 2}$.

Proposition 5.2. The integral of $\exp \left(\frac{1}{2} c_{1}\left(\mathcal{L}_{F}\right)\right)$ over $F$ equals $\left(\# T_{k+c}\right)^{h+r-1}$.

Proof. The line bundle $\mathcal{L}=L(\Sigma)^{2} \otimes K^{-1}$ is $\widehat{L G^{r}}$-equivariant at levels $2(k+c), \ldots, 2(k+c)$. Since $\mathcal{M}(\Sigma)$ carries up to isomorphism a unique line bundle at every level $[29,3.12]$, it follows that $\mathcal{L}$ is the pre-quantum line bundle for the symplectic structure defined by $B_{2(k+c)}$. Hence $\mathcal{L}_{F}$ is a prequantum line bundle for the corresponding symplectic structure on $F$, and $\int_{F} \exp \left(\frac{1}{2} c_{1}\left(\mathcal{L}_{F}\right)\right)$ is the symplectic volume $\operatorname{Vol}_{B_{k+c}}(F)$ for the 2-form defined using $B_{k+c}$. We claim that the symplectic volume coincides with the Riemannian volume, which will complete the proof since $\operatorname{Vol}_{B_{k+c}}\left(T^{2}\right)=\# T_{k+c}$ (see e.g., Beauville [8, Remark 9.9], Bismut-Labourie [10, Prop. 1.2, 1.3]). By our description of $M\left(\Sigma_{h}^{r}\right)$ as a fusion product, the fixed point manifold $F=F\left(\Sigma_{h}^{r}\right)$ is obtained from the fixed point manifold $F\left(\Sigma_{0}^{2}\right)$ (viewed as a group valued Hamiltonian $T^{2}$-space) by fusion: $F\left(\Sigma_{1}^{1}\right)=F\left(\Sigma_{0}^{2}\right)_{\text {fus }}$ and

$$
F\left(\Sigma_{h}^{r}\right)=F\left(\Sigma_{1}^{1}\right) \circledast \cdots \circledast F\left(\Sigma_{1}^{1}\right) \circledast F\left(\Sigma_{0}^{2}\right) \circledast \cdots \circledast F\left(\Sigma_{0}^{2}\right),
$$


with $h$ factors $F\left(\Sigma_{1}^{1}\right)$ and $(r-1)$ factors $F\left(\Sigma_{0}^{2}\right)$. Lemma C.2 from Appendix $\mathrm{C}$ says that the symplectic volume of group valued Hamiltonian torus spaces does not change under fusion. Hence $\operatorname{Vol}_{B_{k+c}}\left(F\left(\Sigma_{h}^{r}\right)\right)=$ $\operatorname{Vol}_{B_{k+c}}\left(F\left(\Sigma_{0}^{2}\right)\right)^{h+r-1}$. Finally, the expression (30) for the 2-form on $M\left(\Sigma_{0}^{2}\right)$ shows that $\operatorname{Vol}_{B_{k+c}}\left(F\left(\Sigma_{0}^{2}\right)\right)$ coincides with the Riemannian volume of $T^{2}$ with respect to $B_{k+c}$.

Proposition 5.3. The phase factor is given by $\zeta_{F}\left(t_{\lambda}\right)^{1 / 2}=(-1)^{(h+r-1) \# \Re_{+}}$.

Proof. The point $m=(e, \ldots, e) \in F$ lies in identity level set of $\Phi$, and its stabilizer in $G^{r}$ is the image of the diagonal embedding of $G$. The 2-form $\omega$ restricts to a symplectic form on the tangent space $E=T_{m} M(\Sigma)$. The factor $\zeta_{F}\left(t_{\lambda}\right)^{1 / 2} \in U(1)$ may be computed using (21).

We first apply this recipe for the 2-holed sphere $\Sigma_{0}^{2}$, so that $E=$ $T_{m} M\left(\Sigma_{0}^{2}\right)=\mathfrak{g} \oplus \mathfrak{g}$. Formula (30) shows that $\omega_{m}$ is the standard 2-form on $\mathfrak{g} \oplus \mathfrak{g}$, given by the inner product $B$. A compatible complex structure is given by the endomorphism $(\xi, \eta) \mapsto(-\eta, \xi)$. Thus, as a complex $G$ representation $E$ is just the complexification $E=\mathfrak{g}^{\mathbb{C}}$. It follows that the eigenvalues of $A$ (other than 1) come in complex conjugate pairs

$$
e^{i \phi_{j}}, e^{-i \phi_{j}}, \quad 0<\phi_{j} \leq \pi / 2
$$

and the corresponding eigenvalues of $A^{1 / 2}$ are $e^{i \phi_{j} / 2}$ and $e^{i \pi-i \phi_{j} / 2}=$ $-e^{-i \phi_{j} / 2}$. Hence

$$
\zeta_{F}\left(t_{\lambda}\right)^{1 / 2}=(-1)^{\# \Re_{+}}
$$

Now consider the case $r \geq 1, h$ arbitrary. The tangent space is $T_{m} M\left(\Sigma_{h}^{r}\right)=(\mathfrak{g} \oplus \mathfrak{g})^{h+r-1}$, but because of the fusion terms the symplectic form is not the standard symplectic form defined by the inner product on $\mathfrak{g}$. However, by Appendix C, Lemma C.3 it is equivariantly and symplectically isotopic to the standard symplectic form. Since the phase factor $\zeta_{F}\left(t_{\lambda}\right)^{1 / 2}$ is a root of unity, it is invariant under equivariant symplectic isotopies, and we conclude as before that $\zeta_{F}\left(t_{\lambda}\right)^{1 / 2}=(-1)^{(h+r-1) \# \Re_{+}}$.

We conclude that the fixed point integral is given by

$$
\chi\left(\nu_{F}, t_{\lambda}\right)=\left(\frac{\# T_{k+c}}{\left|J\left(t_{\lambda}\right)\right|^{2}}\right)^{h+r-1}
$$




\subsection{Verlinde formula.}

From (35) and (32) we obtain:

Theorem 5.4 (Verlinde Formula). Let $G$ be a simply connected Lie group and $k$ a given integral level. The $\operatorname{Spin}_{c}$-index of the moduli space of flat connections on $\Sigma_{h}^{r}$ at level $k$, with markings $\mu=\left(\mu_{1}, \ldots, \mu_{r}\right) \in\left(\Lambda_{k}^{*}\right)^{r}$ is given by the formula

$$
\chi\left(\mathcal{M}\left(\Sigma_{h}^{r}\right)_{\mu}\right)=\left(\# T_{k+c}\right)^{h-1} \sum_{\lambda \in \Lambda_{k}^{*}}\left|J\left(t_{\lambda}\right)\right|^{2-2 h} \chi_{\mu_{1}}\left(t_{\lambda}\right) \cdots \chi_{\mu_{r}}\left(t_{\lambda}\right) .
$$

Remarks 5.5. (a) Theorem (5.4) also covers the case without boundary, since $M\left(\Sigma_{h}^{0}\right)=M\left(\Sigma_{h}^{1}, 0\right)$. One obtains

$$
\chi\left(\mathcal{M}\left(\Sigma_{h}^{0}\right)\right)=\left(\# T_{k+c}\right)^{h-1} \sum_{\lambda \in \Lambda_{k}^{*}}\left|J\left(t_{\lambda}\right)\right|^{2-2 h} .
$$

(b) For the two-holed sphere $\Sigma_{0}^{2}$, formula (36) simplifies by the orthogonality relations for level $k$ characters, and gives $\chi\left(M\left(\Sigma_{0}^{2}\right)_{\mu_{1}, \mu_{2}}\right)=\delta_{\mu_{1}, * \mu_{2}}$.

(c) In Bismut-Labourie [10] the $\operatorname{Spin}_{c}$-indices $\chi\left(\mathcal{M}\left(\Sigma_{h}^{r}\right)_{\mu}\right)$ are computed by direct application of the Kawasaki-Riemann-Roch formula to the reduced spaces. Their approach involves a description of all orbifold strata of the reduced space. The equality with the above sum over level $k$ weights is non-trivial; it is established in [10] for sufficiently high level $k$.

(d) Theorem 5.4 gives a formula for a $\mathrm{Spin}_{c}$-index rather than the dimension of a space of holomorphic sections. Vanishing results for higher cohomology groups have recently been proved by Teleman [34, Section 8].

A more involved computation leads to a formula for semi-simple, compact, connected groups which need not be simply connected, for the case of a single boundary component $r=1$. An interesting feature of our approach is that we obtain a formula for each connected component of the moduli space of flat bundles. We will state the result without proof. Let $G^{\prime}=G / \Gamma$, where $G$ is simply connected and $\Gamma \subset Z(G)$ is a subgroup of the center $Z(G)$ of $G$. For $j=1, \ldots, s$, let $\Gamma_{j} \subset G_{j}$ be the image of $\Gamma$ under projection to the $j$ th simple factor. Let

$$
M^{\prime}\left(\Sigma_{h}^{1}\right)=M\left(\Sigma_{h}^{1}\right) / \Gamma^{2 h}=G^{2 h} / \Gamma^{2 h}=\left(G^{\prime}\right)^{2 h} .
$$


The product of commutators, $\Phi: M\left(\Sigma_{h}^{1}\right)=G^{2 h} \rightarrow G$ is invariant under the action of $\Gamma^{2 h}$ and descends to the $G$-valued moment map $\Phi^{\prime}: M^{\prime}\left(\Sigma_{h}^{1}\right) \rightarrow G$. For any $g^{\prime} \in G^{\prime}$, the moduli space of flat $G^{\prime}$-connections with holonomy conjugate to $g^{\prime}$ is a disjoint union of symplectic quotients $M^{\prime}(\Sigma)_{g}$ where $g$ varies over all pre-images of $g^{\prime}$ in $G$. The reduced spaces at central elements $\gamma \in \Gamma \subset G$ may also be interpreted as moduli spaces of flat connections on the $G^{\prime}$-bundle over $\Sigma_{h}^{0}$, with topological type given by $\gamma$. Let $k=\left(k_{1}, \ldots, k_{s}\right)$, where each $k_{j}$ is a positive multiple of the greatest common divisor of $c_{j}$ and $\# \Gamma_{j}^{2}$. Then there exists a pre-quantum line bundle at level $k$, determined by a choice of character $\phi \in \operatorname{Hom}\left(\Gamma^{2 h}, \mathrm{U}(1)\right)$, and for any $\mu \in \Lambda_{k}^{*}$, the $\operatorname{Spin}_{c}$-index of the symplectic quotient $\mathcal{M}^{\prime}(\Sigma)_{\mu}$ at $\mu$ is given by the formula,

$$
\chi\left(\mathcal{M}^{\prime}(\Sigma)_{\mu}\right)=\frac{\left(\# T_{k+c}\right)^{h-1}}{\# \Gamma^{2 h}} \sum_{\lambda \in \Lambda_{k}^{*}} \epsilon(\phi, \lambda) \frac{\# \Gamma_{\lambda}^{2 h}}{\left|J\left(t_{\lambda}\right)\right|^{2 h-2}} \chi_{\mu}\left(t_{\lambda}\right)^{*} .
$$

Here $\epsilon(\phi, \lambda)=1$ if $\phi$ restricts to the trivial homomorphism on $\Gamma_{\lambda}^{2 h}$, and 0 otherwise. The sum over components leads to a simpler formula, which involves only a sum over weights corresponding to characters of $G^{\prime}$. In the case $G^{\prime}=\operatorname{PSU}(p)$ (5.6) reduces to formulas of Pantev [2'] $(p=2)$, and Beauville $\left[1^{\prime}\right]$ ( $p \geq 3$ prime).

\section{References.}

[1] A. Alekseev, A. Malkin, and E. Meinrenken, Lie group valued moment maps, J. Differential Geom., 48(3) (1998), 445-495.

[2] C. Woodward A. Alekseev, E. Meinrenken. Duistermaat-Heckman measures and moduli spaces of flat bundles over surfaces, to appear in GAFA.

[3] M.F. Atiyah, The geometry and physics of knots, Cambridge University Press, Cambridge, 1990.

[4] M.F. Atiyah and R. Bott, A Lefschetz fixed point formula for elliptic complexes I, Ann. of Math., 86(2) (1967), 374-407.

[5] M.F. Atiyah and R. Bott, A Lefschetz fixed point formula for elliptic complexes II, Applications, Ann. of Math., 88(2) (1968), 451-491.

[6] M.F. Atiyah and G.B. Segal, The index of elliptic operators, II, Ann. of Math., 87(2) (1968), 531-545. 
[7] M.F. Atiyah and I.M. Singer, The index of elliptic operators I, Ann. of Math., 87(2) (1968), 484-530.

[8] A. Beauville, Conformal blocks, fusion rules and the Verlinde formula, in 'Proceedings of the Hirzebruch 65 Conference on Algebraic Geometry' (Ramat Gan, 1993), 9, Israel Math. Conf. Proc., 75-96, Bar-Ilan Univ., Ramat Gan, 1996.

[1'] A. Beauville. The Verlinde formula for PGL(p). In The mathematical beauty of physics (Saclay, 1996), volume 24 of Adv. Ser. Math. Phys., pages 141-151. World Sci. Publishing, 1997.

[9] N. Berline, E. Getzler, and M. Vergne, Heat Kernels and Dirac Operators, Grundlehren der mathematischen Wissenschaften, 298, SpringerVerlag, Berlin-Heidelberg-New York, 1992.

[10] J.M. Bismut and F. Labourie, Symplectic geometry and the Verlinde formulas, in 'Surveys in differential geometry: differential geometry inspired by string theory', 97-311, Int. Press, Boston, MA, 1999.

[11] M. Blau and G. Thompson, Equivariant Kähler geometry and localization in the $G / G$ model, Nuclear Phys. B, 439(1-2) (1995), 367-394.

[12] T. Bröcker and T. tom Dieck, Representations of Compact Lie Groups, Graduate Texts in Mathematics, 98, Springer-Verlag, BerlinHeidelberg-New York, 1985.

[13] S. Chang, Fixed point formula and loop group actions, math. AG/9812148.

[14] S.K. Donaldson, Boundary value problems for Yang-Mills fields, J. Geom. Phys., 8 (1992), 89-122.

[15] J.J. Duistermaat, The Heat Kernel Lefschetz Fixed Point Formula for the Spin-c Dirac Operator, Progress in Nonlinear Differential Equations and Their Applications, 18, Birkhäuser, Boston, 1996.

[16] D.S. Freed. The Verlinde algebra is twisted equivariant K-theory, e-print math.RT/0101038.

[17] D.S. Freed, Classical Chern-Simons theory, I, Adv. Math., 113(2) (1995), 237-303.

[18] A. Gerasimov, Localization in GWZW and Verlinde formula, preprint, Uppsala, 1993, hep-th/9305090. 
[19] V. Guillemin and E. Prato, Heckman, Kostant, and Steinberg formulas for symplectic manifolds, Adv. in Math., 82(2) (1990), 160-179.

[20] V. Guillemin and S. Sternberg, Symplectic Techniques in Physics, Cambridge Univ. Press, Cambridge, 1990.

[21] L.C. Jeffrey and F.C. Kirwan, Intersection pairings in moduli spaces of holomorphic bundles on a Riemann surface, Electron. Res. Announc. Amer. Math. Soc., 1(2) (1995), 57-71 (electronic).

[22] F.C. Kirwan, Convexity properties of the moment mapping, III, Invent. Math., 77 (1984), 547-552.

[23] B. Kostant, Quantization and unitary representations, in C. T. Taam, editor, Lectures in Modern Analysis and Applications III, Lecture Notes in Mathematics, 170, 87-208, Washington, D.C., 1970, SpringerVerlag, Berlin-Heidelberg-New York.

[24] H.B. Lawson, Jr. and M.-L. Michelson, Spin Geometry, Princeton Mathematical Series, 38, Princeton Univ. Press, Princeton, 1989.

[25] E. Lerman and S. Tolman, Hamiltonian torus actions on symplectic orbifolds and toric varieties, Trans. Amer. Math. Soc., 349(10) (1997), 4201-4230.

[26] E. Meinrenken, Symplectic surgery and the Spin ${ }^{\mathrm{c}}$-Dirac operator, Adv. in Math., 134 (1998), 240-277.

[27] E. Meinrenken and R. Sjamaar, Singular reduction and quantization, Topology, 38 (1999), 699-763.

[28] E. Meinrenken and C. Woodward. Canonical bundles for Hamiltonian loop group manifolds, Pacific J. Math., 198(2) (2001), 477-487.

[29] E. Meinrenken and C. Woodward, Cobordism for Hamiltonian loop group actions and flat connections on the punctured two-sphere, Math. Z., 231(1) (1999), 133-168.

[30] E. Meinrenken and C. Woodward, Hamiltonian loop group actions and Verlinde factorization, J. Differential Geom., 50 (1999), 417-470.

[2'] T. Pantev. Comparison of generalized theta functions, Duke Math. J., 76(2) (1994), 509-539. 
[31] A. Pressley and G. Segal, Loop groups, Oxford University Press, Oxford, 1988.

[32] R. Sjamaar and E. Lerman, Stratified symplectic spaces and reduction, Ann. of Math., 134(2) (1991), 375-422.

[33] A. Szenes, The combinatorics of the Verlinde formulas, in 'Vector bundles in algebraic geometry' (Durham, 1993), London Math. Soc. Lecture Note Ser., 208, 241-253, Cambridge Univ. Press, 1995.

[34] C. Teleman, The quantization conjecture revisited, Ann. of Math. (2), $\mathbf{1 5 2}(\mathbf{1})$ (2000), 1-43.

[35] V. Toledano Laredo, Positive energy representations of the loop groups of non-simply connected groups, Comm. Math. Phys., 207 (1999), 307339 .

[36] A. Tsuchiya, K. Ueno, and Y. Yamada, Conformal field theory on universal family of stable curves with gauge symmetries, in 'Integrable systems in quantum field theory and statistical mechanics', Adv. Stud. Pure Math., 19, 459-566, Academic Press, 1989.

[37] M. Vergne, Equivariant index formula for orbifolds, Duke Math. J., 82 (1996), 637-652.

[38] E. Verlinde, Fusion rules and modular transformations in $2 d$ conformal field theory, Nuclear Phys. B, 300 (1988), 360-376.

\author{
UNIVERSITY OF GENEVA \\ SECTION of Mathematics \\ 2-4 RUE DU LIÈVRE \\ 1211 GENÈVE 24 \\ SWITZERLAND \\ E-mail address: alekseev@math.unige.ch \\ UNIVERSITY OF TORONTO \\ DePARTMENT OF MATHEMATICS \\ 100 St George Street \\ Toronto, Ontario M5S3G3 \\ CANADA \\ E-mail address: mein@math.toronto.edu \\ Mathematics-Hill Center \\ RUTGeRs UNIVERSITY
}


110 Frelinghuysen Road

PiscataWAY NJ 08854-8019

E-mail address: ctw@math.rutgers.edu 